\title{
Regional recurrence of oropharyngeal cancer after definitive radiotherapy: a case control study
}

\author{
Karin Söderström ${ }^{1 *}$, Per Nilsson ${ }^{2}$, Tina Dalianis ${ }^{3}$, Elisabeth Kjellén ${ }^{2}$ and Björn Zackrisson ${ }^{1}$
}

\begin{abstract}
Background: Elective treatment of lymph nodes in oropharyngeal cancer (OPC) has impact on both regional recurrences (RR) and risk of late side effects. This study was performed to quantify the dose-dependent impact on RR and overall survival (OS) in a prospectively collected cohort of OPC from the ARTSCAN study with emphasis on elective treatment.

Methods: ARTSCAN is a previously published prospective, randomized, multicentre study of altered radiotherapy (RT) fractionation in head and neck cancer. In ARTSCAN the elective treatment volume for node positive OPC varied significantly between centres due to local treatment principles. All patients with OPC in complete response after primary treatment were eligible for the present case-control study. Cases were patients with RR during five years follow-up. Patients with no recurrence were eligible as controls. Four controls per case were matched according to $T$ - and $N$-stage. Mean $\left(D_{\text {mean }}\right)$ and median $\left(D_{50 \%}\right)$ dose for the lymph node level $(L N L)$ of RR in the cases and the corresponding LNL in the controls were analysed with conditional logistic regression. OS was estimated with the Kaplan-Meier method and evaluated by multivariate Cox regression analysis.
\end{abstract}

Results: There was a dose-dependent risk reduction for $D_{50 \%}$ in the interval that represented elective treatment $(40-50 \mathrm{~Gy})(\mathrm{OR}=0.18, p<0.05)$ and a trend in the same dose interval for $D_{\text {mean }}(\mathrm{OR}=0.19, p=0.07)$. OS rates at five years were $0.39(0.24-0.65)$ for cases and $0.70(0.62-0.81)$ for controls $(p<0.001)$. The Kaplan-Meier and the Cox regression analysis for cases categorised by delivered dose showed an inverse relationship between dose and survival. The cases with RR in a LNL outside planning target volume (PTV) ( $D_{\text {mean }}<40 \mathrm{~Gy}$ ) had an OS rate comparable to that of all patients, and those with RR in a LNL in PTV elective $\left(D_{\text {mean }} 40-60 \mathrm{~Gy}\right)$ or PTV $\mathrm{V}_{\text {tumour }}\left(D_{\text {mean }}>60 \mathrm{~Gy}\right)$ did significantly worse $(p<0.05)$. The same inverse relationship was also shown for a small subset of patient with known HPV-status, defined by over expression of p16 $(p<0.05)$.

Conclusions: There was a significant risk reduction for RR of elective treatment. However the OS for patients with RR outside target volumes was not affected, with similar results for patients with HPV-positive OPC. This could be an argument for a prospective randomized study on limited elective target volumes in OPC.

\section{Background}

Elective radiotherapy (RT) of clinically negative regional lymph nodes has been part of the standard treatment in head and neck squamous cell carcinoma (HNSCC) for many decades $[1,2]$. Several retrospective studies have shown the efficacy of this treatment, but few studies have addressed the dose-response relationships based on the actual dose distribution to the lymph nodes rather than

\footnotetext{
* Correspondence: karin.soderstrom@onkologi.umu.se

'Department of Radiation Sciences, Oncology, Umeå University Hospital,

Umeå University, Umeå, Sweden

Full list of author information is available at the end of the article
}

prescribed dose [3-7]. On the other hand, knowledge about relationships between side effects and doses to organs at risk (OARs) is expanding. A number of models that estimate the probability of normal tissue complications link different dose-volume descriptors for OARs with the incidence of specific side effects, for example dysphagia [8-10].

Since the introduction of three dimensional conformal radiotherapy (3D-CRT) and later intensity modulated radiotherapy (IMRT) and volumetric arc therapy with highly conformal dose distributions, it is possible to better spare OARs to reduce side effects [11-14]. One specific sub-group of HNSCC, oropharyngeal cancer (OPC), 
has undergone a transformation during the last decades. The disease occurs at a younger age and is more often associated with human papilloma virus (HPV) than with tobacco and alcohol abuse [15-19].

As the treatment options evolve and the disease itself changes a reassessment of the current treatment seems motivated.

ARTSCAN was a prospective randomised controlled multi-centre study on accelerated versus conventional fractionation [20]. No significant differences between the fractionation schedules concerning tumour control or late side effects were detected. A large proportion of the patients $(48 \%)$ had OPC. At the time of the study there was no clear national consensus on elective treatment. In the study protocol, the elective target volume was specified as "the standard recommendation at each treating institution" with a dose prescription of 46 Gy in 23 fractions, 5 days per week, in both treatment arms. Some centres practiced limited elective treatment with unilateral and selective (not treating all LNL of the neck) treatment and some used bilateral treatment including all LNL of the neck in node positive disease. There was a significant difference, up to a factor of two, between different study sites regarding the volumes prescribed for elective treatment in node positive OPC [21]. Due to this variation in elective treatment volume, not solely dependent on tumour stage, it is reasonable to use this prospectively collected material to estimate a dose-response relationship for regional recurrences corrected for $\mathrm{T}$ - and $\mathrm{N}$-stage.

Therefore we studied the dose-dependent risk reduction of neck node recurrences as well as survival based on regional recurrences in patients treated with definitive RT in this prospectively collected cohort of OPC.

\section{Methods}

\section{Objectives and study design}

The objectives of the study were:

1. To compare mean and median dose of a lymph node level (LNL) with a regional recurrence to mean and median dose of the corresponding LNL in matched controls without recurrence. This was carried out as a case-control study.

2. To analyse the impact of regional recurrence on overall survival (OS). This was done both for cases vs. controls and for cases grouped according to mean dose separately.

\section{Patients}

The cohort from which the cases and controls were recruited consisted of all OPC patients with complete response to primary treatment in an earlier reported prospective randomised controlled trial, ARTSCAN [20]. Complete response, in this study, was defined as no residual tumour after primary treatment and no local or regional failure within 6 months after randomisation. The ARTSCAN study was approved by the participating centres' local Ethics Committees (Regional ethics committee Umeå) and 733 eligible patients with localised HNSCC signed informed consent between 1998 and 2006. About half of them $(n=357)$ had OPC. Neither chemotherapy nor surgery, other than diagnostic, prior to RT was allowed. Neck dissection as part of primary treatment post RT was allowed according to clinical practice at each participating centre. The use of neck dissection post RT varied between centres where some practiced planned elective neck dissection and some centres used salvage neck dissection in patients with suspected/verified residual neck disease. For additional information on the original study, see [20]. Two of the sites (Stockholm and Umeå) have since the publication of [20] provided information on HPV-status (based on p16-expression as a surrogate marker) for patients with OPC. An extensive quality assurance process for the original study was performed and published [21] with no major trends in the patient characteristics or treatment of OPC during the study period.

Cases for the present study were patients with a regional relapse with or without composite local relapse during follow up. They were identified by the clinical report form and verified in the patient chart. The remaining patients in the cohort with loco-regional control during the follow-up period of five years were eligible as controls. Frequency matching was performed based on $\mathrm{T}$ - and $\mathrm{N}$-stage, and to optimise the statistical power of the study, four controls were chosen for each case.

\section{Radiotherapy}

All patients received computed tomography (CT) based 3D-CRT (92\%) or IMRT (8 \%) with dose prescriptions according to ICRU $[22,23]$. The RT and the quality assurance of the trial have been described in detail elsewhere [20,21]. Patients included in the ARTSCAN trial were randomised between two fractionation schedules for the planning target volume encompassing macroscopic tumour ( $\left.\mathrm{PTV}_{\text {tumour }}\right)$. The conventional fractionation schedule $(\mathrm{CFx})$ consisted of $2 \mathrm{~Gy}$ fractions daily/5 days a week to a total dose of $68 \mathrm{~Gy}$ with a total treatment time $<7$ weeks. Patients receiving accelerated fractionation (AFx) were prescribed 2 Gy fractions daily $/ 5$ days a week plus a daily concomitant boost of 1.1 Gy to a total dose of 68 Gy with a total treatment time $<5$ weeks for $\mathrm{PTV}_{\text {tumour. }}$ As stated in the introduction, all patients had a dose prescription of 46 Gy in 2 Gy fractions daily/5 days a week to the elective PTV $\left(\mathrm{PTV}_{\text {elective }}\right)$, the extent of which was not stipulated in the protocol. The major difference between centres in respect to $\mathrm{PTV}_{\text {elective }}$ was the use of unilateral selective treatment in $\mathrm{T} 1-2$ and $\mathrm{N} 1-2 \mathrm{~b} \mathrm{OPC}$. 


\section{Data collection and localisation of regional relapse}

The patients were clinically examined 4-8 weeks after completion of RT to assess treatment result. During the first two years patients were monitored for loco-regional relapse and distant metastases every 3 months, and they were monitored every 6 months thereafter until five years after end of RT. OS was followed through the Swedish population registry.

Treatment plans (CT-images, structures, plans and dose distribution) from ARTSCAN were collected after RT was completed and stored in DICOM-RT format for additional analysis such as the present.

All cases had their charts revised including available radiological imaging to identify the LNL [24, 25] of the regional relapse including side in respect to the primary tumour (ipsilateral vs. contralateral). The corresponding LNL for the controls including laterality was identified. A systematic delineation of the LNL according to $[26,27]$ was performed by one of the authors (KS) on the original CT-images for all cases and controls. Dose-volume data for these structures were derived from the original DICOM-RT dose files using the software package $R T$ Bench $^{\mathrm{TM}}$ (Cureos AB, Uppsala, Sweden).

\section{Dose-volume analysis}

We analysed mean dose $\left(D_{\text {mean }}\right)$ and median dose $\left(D_{50 \%}\right)$ for the LNL of relapse in the cases and the corresponding LNL in the controls.

The derived doses for both cases and controls were grouped according to delivered dose to the LNL of interest for the calculation of any dose-response relationships. Four dose intervals were chosen representing LNLs: outside PTV (0-40 Gy), within PTV elective (40-50 Gy), in $\mathrm{PTV}_{\text {elective }}$ close to PTV $\mathrm{Pumour}_{\text {(50-60 Gy), and PTV }}$ tumour (>60 Gy), respectively.

\section{Immunohistochemistry of p16}

Tumour biopsy sections $(4-5 \mu \mathrm{m})$ were de-paraffinised and rehydrated, with antigen retrieval in citrate buffer $(\mathrm{pH}$ 6) and unspecific binding sites blocked with $1.5 \%$ horse serum in PBS. The sections were then stained with mAb p16 ${ }^{\text {INKA4a }}$ (clone: JC8, dilution 1:100, Santa Cruz Biotech, Santa Cruz, CA, USA) at $+8{ }^{\circ} \mathrm{C}$ overnight, before incubation for $45 \mathrm{~min}$. with biotinylated anti-mouse antibody (dilution 1:200, Vector Laboratories, Burlingame, CA, USA). Alternatively, the slides were stained with the CINtec $^{\bullet}$ p16 Histology (805-4713), Ventana Medical Systems Inc., Arizona, USA by following the same protocol with the exception of incubation with the antibody for $1 \mathrm{~h}$ at room temperature. For antigen detection, the avidinbiotin-peroxidase complex $(\mathrm{ABC})$ kit (Vectastain, Vector Laboratories, Burlingame, CA, USA) was used. Slides developed in chromogen 3'-diaminobenzydine (DAB) (Vector
Laboratories, Burlingame, CA, USA) and counterstained with haematoxylin were then washed and dehydrated, and the cover mounted using VectaMount permanent mounting media (Vector Laboratories, Burlingame, CA, USA). P16 staining was regarded as positive if $>70 \%$ of the tumour cells were strongly p16-positive $[28,29]$.

\section{Statistical analysis}

For univariate analyses the Pearson Chi-Squared-test and Fisher's exact test were used. The odds ratios for $D_{\text {mean }}$ and $D_{50 \%}$ grouped according to dose intervals were calculated using conditional logistic regression. Clinically suspected interactions with the dose-response data were also evaluated in the model.

The Kaplan-Meier method was used to estimate OS for all patients calculated from start of RT to death or censoring. Univariate survival time comparisons between cases and controls as well as between cases divided by mean dose to LNL of interest were performed using the log-rank test. Kaplan-Meier survival curves were complemented with a multivariate Cox regression, adjusting for potential confounders. All two-way interaction terms were evaluated in the model.

All tests were two-sided and $p$-value of less than 0.05 was considered statistically significant.

Data were analysed with the statistical software package $\mathrm{R}$ (version 2.15.2; R Development Core Team, R foundation for Statistical Computing, Vienna, Austria).

\section{Results}

\section{Eligibility and patient characteristics}

In the cohort of OPC patients with complete response from ARTSCAN, 23 cases with a recurrence in the lymph nodes of the neck were identified corresponding to a regional relapse rate of $7.2 \%$. Five (22 \%) of these regional recurrences had a composite local recurrence. A majority of the cases (95.6\%) and the controls (96.6\%) were in clinical stage III-IV (UICC, Geneva, 1987). $46.6 \%$ of the controls and $43.5 \%$ of the cases were treated with the AFx-schedule. Median age (58 years for cases and 59 years for controls) and other tumour and patient characteristics, including predictive factors reported in [20], showed no significant difference between cases and controls (Table 1). About half of the patients had a neck dissection as part of their primary treatment post RT, and for those the pathology specimen was reviewed for viable tumour (Table 2). In the subset $(n=42)$ with reported HPV-status (based on p16 expression) there was no significant difference between cases and controls (Table 1). In conclusion, none of the tested two-way interactions differed between cases and controls. 
Table 1 Patient and tumour characteristics

\begin{tabular}{|c|c|c|c|c|c|}
\hline & & Controls & Case & Total & \\
\hline & & n (\%) & n (\%) & n (\%) & $p$ value \\
\hline \multirow[t]{5}{*}{$\mathrm{T}$} & 1 & 19 (21.6) & $5(21.7)$ & $24(21.6)$ & 1.000 \\
\hline & 2 & $28(31.8)$ & 7 (30.4) & 35 (31.5) & \\
\hline & 3 & $24(27.3)$ & $6(26.1)$ & $30(27.0)$ & \\
\hline & 4 & 17 (19.3) & $5(21.7)$ & $22(19.8)$ & \\
\hline & Total & 89 (100) & $23(100)$ & $111(100)$ & \\
\hline \multirow[t]{6}{*}{ N } & 0 & $10(11.4)$ & $3(13.0)$ & $13(11.7)$ & 0.069 \\
\hline & 1 & $23(26.1)$ & $2(8.7)$ & $25(22.5)$ & \\
\hline & $2 \mathrm{~A}$ & $25(28.4)$ & $4(17.4)$ & $29(26.1)$ & \\
\hline & $2 \mathrm{~B}$ & $15(17.0)$ & $10(43.5)$ & $25(22.5)$ & \\
\hline & $2 C / 3$ & 15 (17.0) & $4(17.4)$ & 19 (17.1) & \\
\hline & Total & 89 (100) & $23(100)$ & $111(100)$ & \\
\hline \multirow[t]{3}{*}{ Sex } & male & $66(75.0)$ & 19 (82.0) & 85 (76.6) & 0.584 \\
\hline & female & $22(25.0)$ & $4(17.4)$ & 26. (23.4) & \\
\hline & Total & 88 (100) & $23(100)$ & $111(100)$ & \\
\hline \multirow[t]{3}{*}{$\mathrm{Hb}$ conc } & $<140$ & $37(49.3)$ & $14(66.7)$ & $51(53.1)$ & 0.217 \\
\hline & $>140$ & $38(50.7)$ & $7(33.3)$ & 45 (46.9) & \\
\hline & Total & 75 (100) & $21(100)$ & $96(100)$ & \\
\hline \multirow[t]{3}{*}{ PS Karnofsky } & $<90$ & $6(7.2)$ & $3(13)$ & $9(8.5)$ & 0.465 \\
\hline & $>90$ & $77(92,8)$ & $20(87)$ & $97(91.5)$ & \\
\hline & Total & 83 (100) & $23(100)$ & $106(100)$ & \\
\hline \multirow[t]{3}{*}{ HPV-status (>70 \% p16+) } & + & $28(82.4)$ & $6(75.0)$ & $34(81.0)$ & 0.635 \\
\hline & - & $6(17.6)$ & $2(25.0)$ & $8(19.0)$ & \\
\hline & Total & $34(100)$ & $8(100)$ & $42(100)$ & \\
\hline
\end{tabular}

\section{Dose-response relationships}

The dose-response relationships, presented as odds ratios, between cases and controls for the analysed dose intervals including the number of cases and controls analyzed in each interval are presented in Table 3. There was a significant risk reduction for a RR in $D_{50 \%}$ for the interval representing elective treatment (40-50 Gy) and a trend for $D_{\text {mean }}$ in the same dose interval. We have evaluated the model by adjusting for treatment type and neck dissection without any alterations in the significance levels presented in Table 3.

\section{Survival}

OS rates $(95 \% \mathrm{CI})$ at five and twelve years for the cohort were $0.72(0.68-0.77)$ and $0.57(0.5-0.65)$. A RR resulted in significantly reduced OS (Fig. 1). The OS rates at five and twelve years were $0.39(0.24-0.65)$ and 0.29 $(0.15-0.56)$ for the cases and $0.7(0.62-0.81)$ and 0.6 $(0.49-0.74)$ for the controls respectively. The KaplanMeier analysis for all cases categorised by mean dose to LNL with RR (Fig. 2) showed an inverse relationship between dose and survival. The OS rates were significantly higher for cases with a relapse in a LNL outside PTV

Table 2 Neck dissection (ND) as part of primary treatment after definitive RT

\begin{tabular}{|c|c|c|c|c|c|}
\hline & & Control & Case & Total & $p$ value \\
\hline & & n (\%) & n (\%) & n (\%) & \\
\hline \multirow[t]{3}{*}{ ND } & Yes & $54(61.4)$ & $11(47.8)$ & $65(58.6)$ & 0.342 \\
\hline & No & 34 (38.6) & $12(52.2)$ & $46(41.4)$ & \\
\hline & Total & $88(100)$ & $23(100)$ & $111(100)$ & \\
\hline \multirow[t]{3}{*}{ If ND, viable tumour present } & Yes & $15(27.8)$ & $3(27.3)$ & $18(28.1)$ & 0.219 \\
\hline & No & $39(72.2)$ & $7(63.6)$ & $46(71.2)$ & \\
\hline & Total & $54(100)$ & $10(100)$ & $64(100)$ & \\
\hline
\end{tabular}


Table 3 Dose-response relationship

\begin{tabular}{|c|c|c|}
\hline Dose interval & OR & \multirow[t]{2}{*}{$\begin{array}{l}p \\
\text { value }\end{array}$} \\
\hline No of RR/controls & & \\
\hline \multicolumn{3}{|l|}{ Mean dose, $D_{\text {mean }}$} \\
\hline $10-40$ & 0.85 & 0.856 \\
\hline \multicolumn{3}{|l|}{$5 / 9$} \\
\hline $40-50$ & 0.19 & 0.073 \\
\hline \multicolumn{3}{|l|}{$3 / 27$} \\
\hline $50-60$ & 0.33 & 0.326 \\
\hline \multicolumn{3}{|l|}{$2 / 9$} \\
\hline$>60$ & 0.48 & 0.453 \\
\hline \multicolumn{3}{|l|}{$9 / 29$} \\
\hline \multicolumn{3}{|c|}{ Reference $=$ dose category $0-10$} \\
\hline \multicolumn{3}{|l|}{$4 / 6$} \\
\hline \multicolumn{3}{|l|}{ Median dose, $D_{50 \%}$} \\
\hline $10-40$ & 0.93 & 0.935 \\
\hline \multicolumn{3}{|l|}{$4 / 7$} \\
\hline $40-50$ & 0.18 & 0.049 \\
\hline \multicolumn{3}{|l|}{$3 / 34$} \\
\hline $50-60$ & 1.57 & 0.705 \\
\hline \multicolumn{3}{|l|}{$2 / 2$} \\
\hline$>609 / 31$ & 0.65 & 0.650 \\
\hline \multicolumn{3}{|c|}{ Reference $=$ dose category 0-10 } \\
\hline $4 / 6$ & & \\
\hline
\end{tabular}

$\left(D_{\text {mean }}<40 \mathrm{~Gy}\right)(0.67(0.42-1.00))$ than for those with a relapse in a LNL in the elective volume ( $\left.D_{\text {mean }} 40-60 \mathrm{~Gy}\right)$ (0.40 (0.14-1.00) and in PTV tumour $\left(D_{\text {mean }}>60\right.$ Gy) $(0.11$ (0.02-0.71). A similar relative inverse relationship was shown for the more limited number of cases with known HPV-status (by p16 over expression in $>70 \%$ of the cells) divided in two groups according to $D_{\text {mean }}$. OS rate at five years was $0.5(0.25-1.00)$ for $D_{\text {mean }}<60$ Gy and none were alive at 5 years for $D_{\text {mean }}>60$ Gy $(p=0.046)$.

Complementing the results presented in Fig. 1, we performed a Cox regression with OS as dependent variable, adjusting for fractionation schedule (AFx vs. CFx) and neck surgery. The variables representing time to relapse and neck surgery were analyzed as a time-dependent (i.e. time at risk is counted from the day of the event until either end of follow up or death). The results are presented in Table 4. No tested two-way interactions were statistically significant. When comparing cases and controls and not taking date of recurrence into account (i.e. not analyzing recurrence as a time-dependent variable) the hazard ratio for cases vs. controls was 2.74
(95\% CI 1.44-5.21). A Cox regression analysis was also performed for cases only, with outcome OS complementing Fig. 2, comparing dose categories ( $\leq 40$ Gy, $40 \leq$ $60 \mathrm{~Gy},>60 \mathrm{~Gy}$ ) adjusted for fractionation schedule and neck surgery (analyzed as a time-dependent variable), Table 5. No tested two-way interactions were statistically significant.

\section{Discussion}

Our material indicates a risk reduction for regional recurrences $(\mathrm{OR}<0.2)$ in the dose interval corresponding to elective treatment (40-50 Gy) with statistical significance in the logistic regression model for $D_{50 \%}$ and a trend for $D_{\text {mean }}$. No significant risk reduction for regional relapse was found in our study for $D_{\text {mean }}$ and $D_{50 \%}$ in the other investigated dose intervals. One explanation for the lack of risk reduction for development of RR in $D_{50 \%}$ and $D_{\text {mean }}>50$ Gy could be the close proximity to macroscopic tumour in these LNLs and thereby an inherent higher risk of relapse where a dose $<60$ Gy might not be sufficient. However, the number of cases and controls in this dose interval is small. A similar dose as suggested in our material has been proposed for elective treatment to the neck in recent studies where an equivalent dose in 2 Gy fractions, assuming $\alpha$ / $\beta=10$ Gy $\left(\right.$ EQD2 $\left._{10}\right)>50 \mathrm{~Gy}$, does not seem to improve regional control [30, 31], whereas a dose $<30$ Gy in 2 Gy fractions has been shown to have a higher regional relapse rate [32]. A prospective randomised trial investigating an EQD2 10 of 40 Gy to $\mathrm{PTV}_{\text {elective }}$ presented encouraging toxicity outcome regarding dysphagia, but not yet tumour control data [33].

As the cohort for the present study consisted of patients with complete response after primary treatment, the OS of the cohort was somewhat better than the survival of all OPS presented in [20]. In our study a regional relapse resulted in significantly reduced OS, both at five and twelve years. However, when deciding which LNLs to include in an elective target volume, the consequence on OS of a nodal recurrence outside of the target volume is highly relevant. The effect on OS of regional relapses categorised by delivered dose, however, is sparsely addressed in the literature. This prompted us to do a survival analysis for the cases grouped according to $D_{\text {mean }}$ representing a relapse outside of the PTV $(<40$ Gy), in the PTV elective (40$60 \mathrm{~Gy})$, or in the $\mathrm{PTV}_{\text {tumour }}(>60 \mathrm{~Gy})$. A recurrence in PTV $_{\text {tumour }}$ has a major impact on OS, whereas a patient with a nodal relapse outside the PTVs has a survival comparable to that of the cohort. Our hypothesis, to this perhaps somewhat contra intuitive finding, is that the relapses in such heavily treated areas are selected to be more treatment resistant than a recurrence in a 


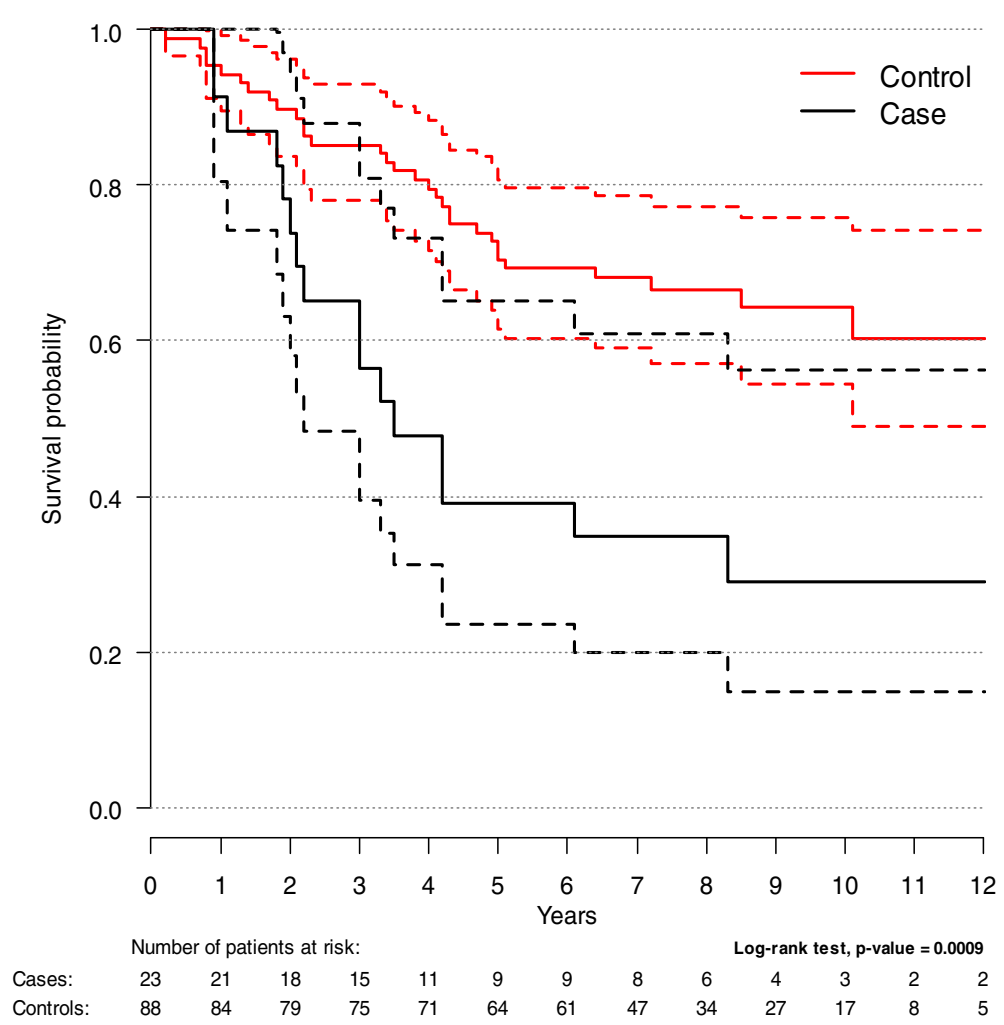

Fig. 1 Overall survival in cases and controls. Kaplan-Meier plot of overall survival in cases and controls (95\% Cl)

treatment naive LNL, as well as having more limited treatment options.

The finding that recurrences "out of field" have a survival comparable to the cohort could be an argument for future studies investigating limited elective target volumes omitting treatment to LNL with low risk of relapse in order to avoid late side effects of the treatment.

Since HPV is one of the most important prognostic factors in OPC treated with RT [34-37], we evaluated the OS in the cases with HPV-positive tumours divided according to $D_{\text {mean }}$ to examine if the inverse relationship between dose and survival seen in all cases holds true for the HPVpositive cases as well. Because of the small number of cases with known HPV-status we restricted the analysis to $D_{\text {mean }} \leq 60$ Gy or $D_{\text {mean }}>60$ Gy in this group. In spite of the small number of cases there was still a significant difference in OS in patients with HPV-positive OPC divided according to dose similar to that shown for all cases. Recently a review of risk factors for loco-regional failure in HPV-associated OPC found that the only factors significant for risk stratification in multivariate analysis apart from smoking were T4- and N3-stages [38], which were evenly distributed in our study between cases and controls. Only five of the HPV-positive cases had available smoking status (all non-smokers). Three of those had $D_{\text {mean }} \leq 60$ Gy and two had $D_{\text {mean }}>60$.
Two different fractionation schedules were used for the PTV $_{\text {tumour }}$ in the present study. The ARTSCAN study showed no significant difference in treatment response of lymph node metastases or regional relapses between the fractionation schedules and the use of AFx in our study. Thus the study was well balanced between cases and controls. Therefore no significant difference in outcome depending on fractionation schedule was expected.

Today advanced head and neck cancer is commonly treated with chemo-RT which has shown a survival benefit compared to RT alone [39]. The presented doseresponse relationships in this study are investigated for RT without chemotherapy. Since HPV-associated OPC has a favourable outcome, voices are being raised for a possible de-escalation of the treatment to this entity of HNSCC [18, 37, 40]. The incidence of HPV-associated OPC is steadily increasing and now constitutes up to $85 \%$ of all OPC in Sweden $[16,17]$. Therefore we suggest that dose-response relationships for RT without chemotherapy are clinically relevant.

According to the ARTSCAN-study protocol, the PTV elective was defined according to clinical practice at each participating centre. At the time of the study there was no clear consensus on what to treat electively in Sweden. This resulted in an up to two- fold variation in the size of

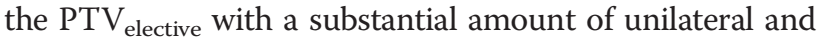




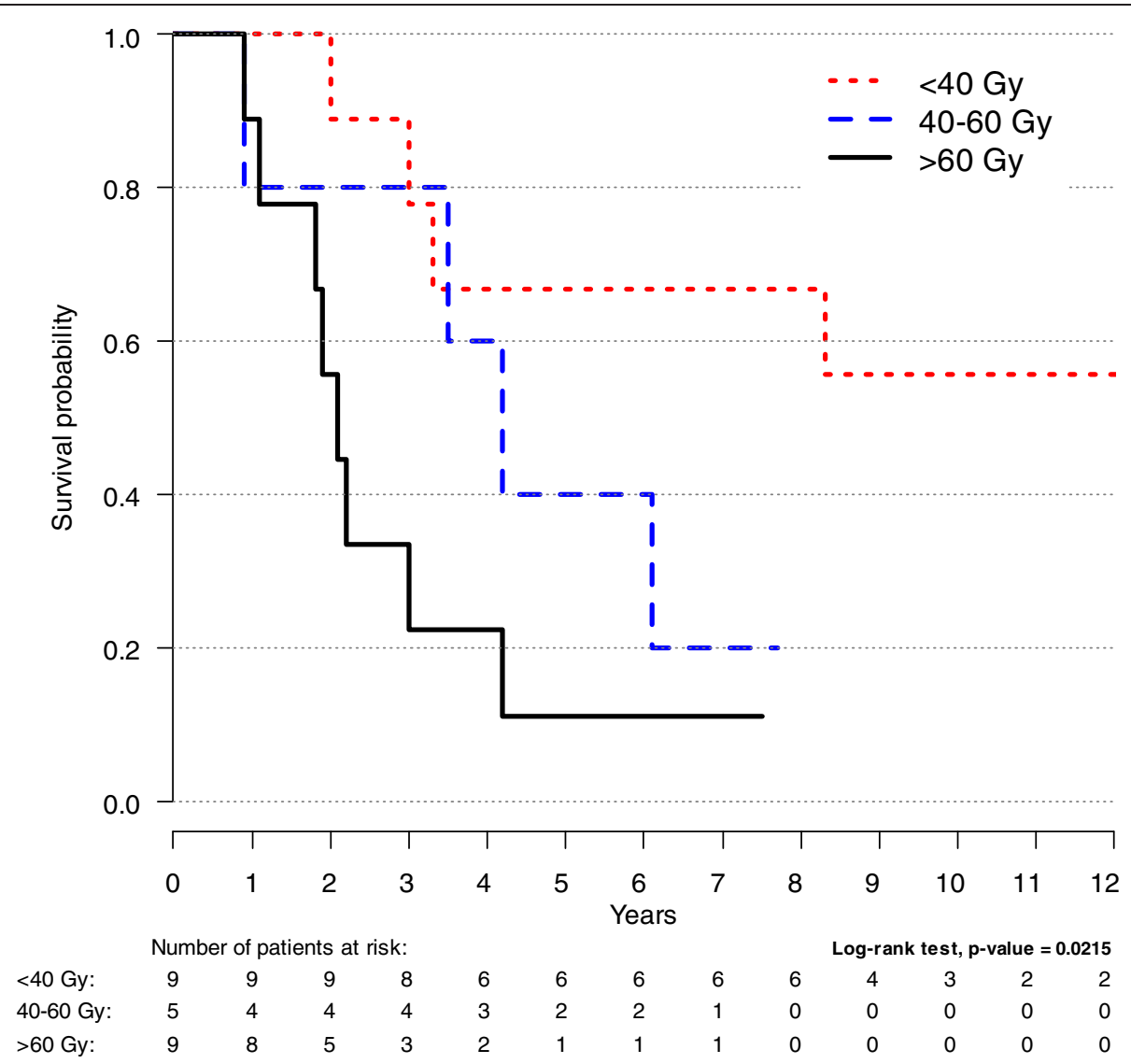

Fig. 2 Overall survival in cases based on mean dose $\left(D_{\text {mean }}\right)$. Kaplan-Meier plot of overall survival in cases divided according to mean dose to $L N L$ of interest

selective elective treatment in the study. Therefore the variation of dose delivered to the LNLs of the neck was only partly dependent on $\mathrm{T}$ - and $\mathrm{N}$-stage. This allows analysis of dose-response relationships of regional recurrences corrected for tumour stage. Moreover, the present study was performed on a patient material consisting solely of OPC, which reduces the possible bias of different risk of metastatic spread with respect to primary tumour site.

\section{Conclusions}

Our study shows a significant risk reduction for the development of regional recurrences of elective treatment

Table 4 Overall survival in cases and controls

\begin{tabular}{llll}
\hline & $\mathrm{HR}$ & $\mathrm{Cl}$ & $p$-value \\
\hline Regional relapse & 3.56 & $1.84-6.92$ & $<0.001$ \\
Fx-schedule & 0.88 & $0.49-1.59$ & 0.673 \\
Neck dissection & 0.85 & $0.45-1.60$ & 0.619 \\
\hline
\end{tabular}

Cox regression analysis of overall survival in cases vs. controls adjusted for fractionation schedule and neck dissection in the dose levels used in the present study compared to no prescribed elective treatment in OPC. However, in 319 patients treated with RT without chemotherapy, only 23 nodal recurrences occurred during five years of follow-up. Moreover the OS for patients with regional recurrence outside target volumes was not affected, with a similar pattern for a small subset of patients with known HPV-status. This could be an argument for a prospective randomised study on limited elective target volumes to reduce late side effects in OPC.

Table 5 Overall survival in cases based on mean dose $\left(D_{\text {mean }}\right)$

\begin{tabular}{llll}
\hline & HR & $\mathrm{Cl}$ & $p$-value \\
\hline $40-60$ & 1.67 & $0.35-7.98$ & 0.522 \\
$60-80$ & 7.94 & $1.80-35.04$ & 0.006 \\
Fx-schedule & 1.96 & $0.54-7.17$ & 0.310 \\
Neck dissection & 1.83 & $0.59-5.67$ & 0.298 \\
\hline
\end{tabular}

Cox regression analysis of overall survival in cases categorised according to dose interval in Gy adjusted for fractionation schedule and neck dissection. Dose $<40 \mathrm{~Gy}$ is the reference category 


\section{Competing interests}

The authors declare that they have no competing interests.

\section{Authors' contributions}

BZ and KS were involved in the study design (BZ, EK and PN for the initial ARTSCAN trial design); PN and KS was responsible for deriving the dose volume parameters; TD was responsible for the analysis of p16, KS was responsible for writing the manuscript; and PN, EK, TD and BZ were responsible for critical revision of the manuscript. All authors read and approved the final version of the manuscript.

\section{Acknowledgements}

This work was supported by grants from the Swedish Cancer Society and the Cancer Research Foundation of Northern Sweden, the Stockholm Cancer Society and Cancer and Allergy Foundation. The study sponsors had no role in the study design, data collection, data analysis, writing, or submission of the manuscript. This study was made possible by the commitment from the staff at participating centres in the ARTSCAN study: Umeå University Hospital; Lund University Hospital; Malmö University Hospital; Karolinska University Hospital, Stockholm; Örebro University Hospital; Karlstad Central Hospital; Sahlgrenska University Hospital, Göteborg; Linköping University Hospital; Gävle Hospital; Ryhov County Hospital, Jönköping; and Uppsala University Hospital.

The authors acknowledge Ove Björ, Regional Cancer Centre, Umeå, for statistical advice and analysis.

\section{Author details}

'Department of Radiation Sciences, Oncology, Umeå University Hospital, Umeå University, Umeå, Sweden. ${ }^{2}$ Department of Oncology and Radiation Physics, Skåne University Hospital, Lund University, Lund, Sweden. ${ }^{3}$ Department of Oncology-Pathology, Karolinska Institutet, Karolinska University Hospital, Solna, Sweden.

\section{Received: 21 January 2015 Accepted: 15 May 2015}

Published online: 27 May 2015

\section{References}

1. Fletcher $\mathrm{GH}$, Lindberg RD. Squamous cell carcinomas of the tonsillar area and palatine arch. Am J Roentgenol Radium Ther Nucl Med. 1966;96:574-87.

2. Fletcher $\mathrm{GH}$. Elective irradiation of subclinical disease in cancers of the head and neck. Cancer. 1972;29:1450-4.

3. Rabuzzi DD, Chung CT, Sagerman RH. Prophylactic neck irradiation. Arch Otolaryngol (Chicago, III : 1960). 1980;106:454-5.

4. Mendenhall WM, Million RR, Cassisi NJ. Elective neck irradiation in squamouscell carcinoma of the head and neck. Head Neck Surg. 1980;3:15-20.

5. Mendenhall WM, Parsons JT, Million RR. Elective lower neck irradiation: 5000 cGy/25 fractions versus 4050 cGy/15 fractions. Int J Radiat Oncol Biol Phys. 1988;15:439-40.

6. August M, Gianetti K. Elective neck irradiation versus observation of the clinically negative neck of patients with oral cancer. J Oral Maxillofac Surg Off J Am Assoc Oral Maxillofac Surg. 1996;54:1050-5.

7. Boysen M, Lovdal O, Soberg R, Jacobsen AB, Tausjo J, Evensen JF. Elective radiotherapy of the neck in patients with squamous cell carcinoma of the head and neck. ORL; J Oto-Rhino-Laryngol and its Related Specialties. 1992;54:103-7.

8. Christianen ME, Schilstra C, Beetz I, Muijs CT, Chouvalova O, Burlage FR, et al. Predictive modelling for swallowing dysfunction after primary (chemo) radiation: results of a prospective observational study. Radiother Oncol J Eur Soc Ther Radiol Oncol. 2012;105:107-14.

9. Eisbruch A, Kim HM, Feng FY, Lyden TH, Haxer MJ, Feng M, et al. Chemo-IMRT of oropharyngeal cancer aiming to reduce dysphagia: swallowing organs late complication probabilities and dosimetric correlates. Int J Radiat Oncol Biol Phys. 2011;81:e93-9.

10. Mortensen HR, Jensen K, Aksglaede K, Behrens M, Grau C. Late dysphagia after IMRT for head and neck cancer and correlation with dose-volume parameters. Radiother Oncol J Eur Soc Ther Radiol Oncol. 2013;107:288-94.

11. Dirix $P$, Nuyts $S$. Evidence-based organ-sparing radiotherapy in head and neck cancer. Lancet Oncol. 2010;11:85-91.

12. Doornaert P, Verbakel WF, Rietveld DH, Slotman BJ, Senan S. Sparing the contralateral submandibular gland without compromising PTV coverage by using volumetric modulated arc therapy. Radiat Oncol (London, England). 2011;6:74.

13. Eisbruch A, Levendag PC, Feng FY, Teguh D, Lyden T, Schmitz PI, et al. Can IMRT or brachytherapy reduce dysphagia associated with

chemoradiotherapy of head and neck cancer? The Michigan and Rotterdam experiences. Int J Radiat Oncol Biol Phys. 2007;69:S40-2.

14. Eisbruch A, Schwartz M, Rasch C, Vineberg K, Damen E, Van As CJ, et al. Dysphagia and aspiration after chemoradiotherapy for head-and-neck cancer: which anatomic structures are affected and can they be spared by IMRT? Int J Radiat Oncol Biol Phys. 2004;60:1425-39.

15. Marur S, D'Souza G, Westra WH, Forastiere AA. HPV-associated head and neck cancer: a virus-related cancer epidemic. Lancet Oncol. 2010;11:781-9.

16. Nasman A, Attner P, Hammarstedt L, Du J, Eriksson M, Giraud G, et al. Incidence of human papillomavirus (HPV) positive tonsillar carcinoma in Stockholm, Sweden: an epidemic of viral-induced carcinoma? Int J Canc J Int Canc. 2009;125:362-6.

17. Attner P, Du J, Nasman A, Hammarstedt L, Ramqvist T, Lindholm J, et al. The role of human papillomavirus in the increased incidence of base of tongue cancer. Int J Canc J Int Canc. 2010;126:2879-84.

18. Ramqvist T, Dalianis T. An epidemic of oropharyngeal squamous cell carcinoma (OSCC) due to human papillomavirus (HPV) infection and aspects of treatment and prevention. Anticancer Res. 2011;31:1515-9.

19. Chaturvedi AK, Anderson WF, Lortet-Tieulent J, Curado MP, Ferlay J, Franceschi S, et al. Worldwide trends in incidence rates for oral cavity and oropharyngeal cancers. J Clin Oncol Off J Am Soc Clin Oncol. 2013;31:4550-9.

20. Zackrisson B, Nilsson P, Kjellen E, Johansson KA, Modig H, Brun E, et al. Two-year results from a Swedish study on conventional versus accelerated radiotherapy in head and neck squamous cell carcinoma-the ARTSCAN study. Radiother Oncol J Eur Soc Ther Radiol Oncol. 2011;100:41-8.

21. Johansson KA, Nilsson P, Zackrisson B, Ohlson B, Kjellen E, Mercke C, et al. The quality assurance process for the ARTSCAN head and neck study - a practical interactive approach for QA in 3DCRT and IMRT. Radiother Oncol J Eur SocTher Radiol Oncol. 2008;87:290-9.

22. International Commision of Radiation Units and Measurements, ICRU report 50: Prescribing, recording, and reporting photon-beam therapy. Bethesda; 1993. ICRU;1993 (ISBN 0-913394-48-3).

23. International Commision of Radiation Units and Measurements, ICRU report 62: Prescribing, recording, and reporting photon-beam therapy (supplement to ICRU report 50). Bethesda; 1999. ICRU;1999 (ISBN 0-913394-61-0).

24. Robbins KT, Medina JE, Wolfe GT, Levine PA, Sessions RB, Pruet CW. Standardizing neck dissection terminology. Official report of the Academy's Committee for Head and Neck Surgery and Oncology. Arch Otolaryngol Head Neck Surg. 1991;117:601-5.

25. Gregoire V, Coche E, Cosnard G, Hamoir M, Reychler H. Selection and delineation of lymph node target volumes in head and neck conformal radiotherapy. Proposal for standardizing terminology and procedure based on the surgical experience. Radiother Oncol J Eur SocTher Radiol Oncol. 2000;56:135-50.

26. Gregoire V, Eisbruch A, Hamoir M, Levendag P. Proposal for the delineation of the nodal CTV in the node-positive and the post-operative neck. Radiother Oncol J Eur SocTher Radiol Oncol. 2006;79:15-20.

27. Gregoire V, Levendag P, Ang KK, Bernier J, Braaksma M, Budach V, et al. CT-based delineation of lymph node levels and related CTVS in the node-negative neck: DAHANCA, EORTC, GORTEC, NCIC, RTOG consensus guidelines. Radiother Oncol J Eur SocTher Radiol Oncol. 2003;69:227-36.

28. Ang KK, Harris J, Wheeler R, Weber R, Rosenthal DI, Nguyen-Tan PF, et al. Human papillomavirus and survival of patients with oropharyngeal cancer. N Engl J Med. 2010;363:24-35.

29. Begum S, Gillison ML, Ansari-Lari MA, Shah K, Westra WH. Detection of human papillomavirus in cervical lymph nodes: a highly effective strategy for localizing site of tumor origin. Clin Canc Res Off J Am Assoc Canc Res. 2003;9:6469-75.

30. Duprez F, Bonte K, De Neve W, Boterberg T, De Gersem W, Madani I. Regional relapse after intensity-modulated radiotherapy for head-and-neck cancer. Int J Radiat Oncol Biol Phys. 2011;79:450-8.

31. Dandekar V, Morgan T, Turian J, Fidler MJ, Showel J, Nielsen T, et al. Patterns-of-failure after helical tomotherapy-based chemoradiotherapy for head and neck cancer: implications for CTV margin, elective nodal dose and bilateral parotid sparing. Oral Oncol. 2014;50:520-6.

32. Suwinski R, Maciejewski B, Withers HR. Dose-response relationship for elective neck irradiation of head and neck cancer-facts and controversies. Neoplasma. 1998;45:107-12. 
33. Nuyts S, Lambrecht M, Duprez F, Daisne JF, Van Gestel D, Van den Weyngaert $\mathrm{D}$, et al. Reduction of the dose to the elective neck in head and neck squamous cell carcinoma, a randomized clinical trial using intensity modulated radiotherapy (IMRT). Dosimetrical analysis and effect on acute toxicity. Radiother Oncol J Eur SocTher Radiol Oncol. 2013;109:323-9.

34. Lassen P, Eriksen JG, Krogdahl A, Therkildsen MH, Ulhoi BP, Overgaard M et al. The influence of HPV-associated p16-expression on accelerated fractionated radiotherapy in head and neck cancer: evaluation of the randomised DAHANCA 6\&7 trial. Radiother Oncol J Eur SocTher Radiol Oncol. 2011;100:49-55.

35. Lassen P. The role of Human papillomavirus in head and neck cancer and the impact on radiotherapy outcome. Radiother Oncol I Eur SocTher Radiol Oncol. 2010:95:371-80.

36. Rischin $D$, Young RJ, Fisher R, Fox SB, Le QT, Peters $L$, et al. Prognostic significance of p16INK4A and human papillomavirus in patients with oropharyngeal cancer treated on TROG 02.02 phase III trial. J Clin Oncol Off J Am Soc Clin Oncol. 2010;28:4142-8.

37. Ang KK, Sturgis EM. Human papillomavirus as a marker of the natural history and response to therapy of head and neck squamous cell carcinoma. Semin Radiat Oncol. 2012;22:128-42.

38. Vainshtein JM, Spector ME, McHugh JB, Wong KK, Walline HM, Byrd SA, et al. Refining risk stratification for locoregional failure after chemoradiotherapy in human papillomavirus-associated oropharyngeal cancer. Oral Oncol. 2014;50:513-9.

39. Blanchard P, Baujat B, Holostenco V, Bourredjem A, Baey C, Bourhis J, et al. Meta-analysis of chemotherapy in head and neck cancer (MACH-NC): a comprehensive analysis by tumour site. Radiother Oncol J Eur SocTher Radiol Oncol. 2011:100:33-40.

40. Sturgis EM, Ang KK. The epidemic of HPV-associated oropharyngeal cancer is here: is it time to change our treatment paradigms? J Natl Compr Canc Netw. 2011:9:665-73.

\section{Submit your next manuscript to BioMed Central and take full advantage of:}

- Convenient online submission

- Thorough peer review

- No space constraints or color figure charges

- Immediate publication on acceptance

- Inclusion in PubMed, CAS, Scopus and Google Scholar

- Research which is freely available for redistribution 